\title{
Outcome and complications of Ventralex mesh repair in umbilical hernia
}

\author{
Krisna Rani Majumder, Md Rassell, Hasan Shahrear Ahmed, Shaila Parveen
}

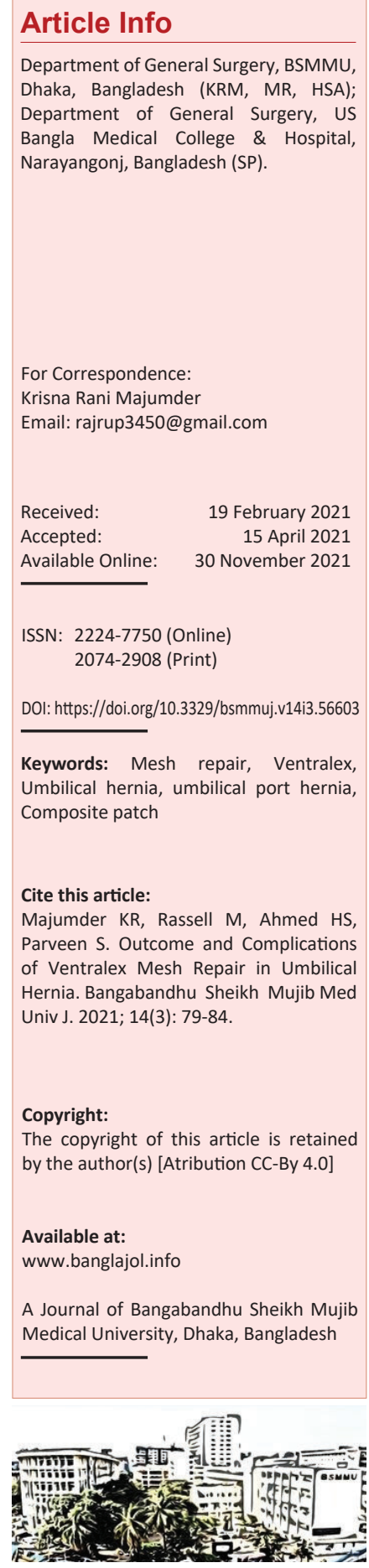

\begin{abstract}
In recent time, various techniques have been applied for the hernia repair surgery. Considering the possibility of the recurrence rates up to $40 \%$, umbilical and umbilical port hernias have been repaired without mesh for a long time. The repair of these hernias by the Ventralex hernia patch may decrease recurrent hernia rates. The aim of this study was to evaluate the outcome and complications of mesh repair in umbilical hernia by the Ventralex hernia patch. A total of 36 patients were studied retrospectively who underwent mesh repair surgery by the Ventralex hernia patch between April 2017 and October 2019 at Anower Khan Modern Medical College \& Hospital, Care Medical College \& Hospital and LABAID Specialized Hospital. They have also been evaluated for instant post-operative complications, recurrence rate \& pain. Short-term clinical outcomes were assessed during post-operative follow up at first and sixth week. One year long-term outcomes were assessed over telephone. In this study, there were 28 female and 8 male patients with the range of age from $24-68$ years (Mean $41.5 \pm 8.36$ ) having 27 umbilical hernias and 9 umbilical port hernias. No major short-term complications were found but early minor complications were found in 5 patients $(13.89 \%)$. No pain or mild pain was reported in 31 patients after one week's post-operative follow up. 34 patients reported being very satisfied with their repairs. In this study we found that the Ventralex hernia patch is an effective and easier technique for mesh repair in umbilical and umbilical port hernias. This technique can also save the operative time with less post-operative complications and better outcomes.
\end{abstract}

\section{Introduction}

Various studies were described that the mesh repairing of the ventral hernias has reduced the complexity and recurrence rates significantly. ${ }^{1,2}$ However, generally the mesh repairing indicates a more extensive dissection that might higher the morbidity. ${ }^{3}$ Traditional repair techniques without mesh are associated with high recurrence rates, which seems to be an overkill for a small umbilical hernia with $<3 \mathrm{~cm}$ gap. In regards to the larger defects $(>3 \mathrm{~cm}$ in diameter), hernioplasty with mesh and pre- peritoneal technique have been popular for their lower recurrence rate, decreased postoperative pain, and faster recovery. ${ }^{4-6}$ Evidences show that a large-sized Ventralex ${ }^{\mathrm{TM}}$ patch should preferably not be inserted intraperitoneally. In spite of, it is not suggested intially by the
Bard ${ }^{\circledR}$ company, in this series we have systematically closed the fascia. Mesh repair should be considered even in these smaller hernias because most of the cases, primary closure often fails and these hernias are prone to complications. ${ }^{8-10}$ Umbilical and umbilical port hernias are still among the common surgical challenge. About $6 \%-14 \%$ of abdominal wall surgeries are affected by umbilical hernias. ${ }^{(11)}$ Repair mesh of the ventral hernia is still now a common operation performed by the surgeons all over the world. ${ }^{12} 10-15 \%$ of all primary hernias are ventral hernias and this hernias include umbilical, epigastric, and incisional hernias. ${ }^{13}$ This hernias are often symptomatic with the risk of incarceration due to the adhesion of omentum to the hernial sac. This is why, most umbilical hernias require surgical repair. ${ }^{14}$ Now a days, most of the surgeons suggest for 
the surgical repair of the ventral hernias to avoid the risk of incarceration and strangulation. ${ }^{15}$ Multiple surgical techniques have been described for mesh repair including onlay, sublay, and inlay. ${ }^{16}$ In the case of traditional suture-repair techniques, there have been a recurrence rates of up to $50 \%$ and this have led to the more frequent use of prosthetic meshes, especially in defects $>3 \mathrm{~cm} .{ }^{(17-19}$ The ventralex ${ }^{\mathrm{TM}}$ hernia patch $(($ Bard $\AA$, Davol, Warwick, RI) is a self-expanding, non-absorbable, and circular bilayer prosthesis. It has an outer polypropylene monofilament mesh and an inner expanded polytetrafluoroethylene (ePTFE) surface. ${ }^{20}$ There are several benefits of this technique and the main benefit is to fix mesh that is achieved principally by the intra-abdominal pressure. It holds the prosthesis against the deep surface of the muscle and as well as potentially improving tissue integration into the polypropylene side of the mesh. The short-term clinical outcome has been promising. ${ }^{21-23}$ but in the cases of the long-term outcome, the recurrence and complication rates have been equivocal in a small number of studies. ${ }^{23-26}$ In this study, our aim was to evaluate the the outcome and complications of mesh repair in umbilical hernia by the Ventralex ${ }^{\mathrm{TM}}$ hernia patch.

\section{Methods}

Data were collected retrospectively of 36 patients with umbilical hernia ( 27 patients) and umbilical port hernia ( 9 patients) who had a defect size $1-3 \mathrm{~cm}$ in diameter and repaired using an open underlay (or sublay) technique with the Ventralex $^{\mathrm{TM}}$ patch from between April 2017 and October 2019 at Anower Khan Modern Medical College \& Hospital, Care Medical College \& Hospital and LABAID Specialized Hospital. The trial was seen as a quality control of a cohort of patients treated with an innovative mesh device. All the patients were informed about taking part in this study. Total number of patients was 36. Data was collected regarding early mesh related complication like pain, infection, late complications like recurrence at 12 months, Quality of life, and overall satisfaction.

\section{Surgical Procedure}

All Ventralex mesh repairs were carried out with the patient in the supine position by single surgeon under general or spinal anesthesia using mechanical ventilation by laryngeal mask airway, a small infraumbilical curvilinear skin incision was made. Over hernia, the skin was incised transversely. Then the hernial sac was dissected out, opened, and excised when it's necessary after reduction of it's contents. (Figure-1) The prosthesis was pre-soaked in gentamicin, folded in half to allow entry through the small gap, inserted through the defect and positioned intraperitonealy such that the ePTFE side faces the peritoneal cavity and PP side faces the parietal wall.(Figure-2) A medium $(6.4 \mathrm{~cm})$ or large $(8 \mathrm{~cm})$-sized Ventralex ${ }^{\mathrm{TM}}$ mesh was deployed and the straps were secured onto the defect edges with 2-4 interrupted 2/0 Prolene TM (CEthicon, Cincinnati, Ohio, USA) ensuring they were not too tight to avoid a cupping effect of the mesh. Then the fascia defect was approximated anteriorly using interrupted sutures of 1-PDS (polydioxanone suture).(Figure-3) By the interrupted intradermal absorbable suture, the Skin was closed and then a water proof dressing was applied and kept undisturbed intact for 5 days. Patients were discharged home on first post-operative day, on oral antibiotic for 5 days and simple analgesia as required.

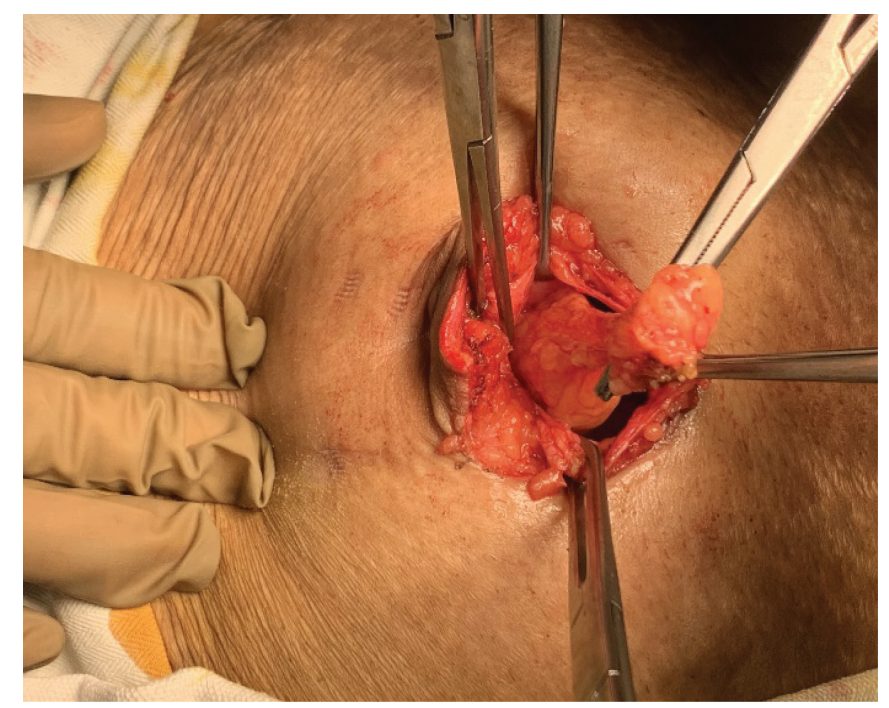

Figure-1 Dissection of the hernia sac and it's content

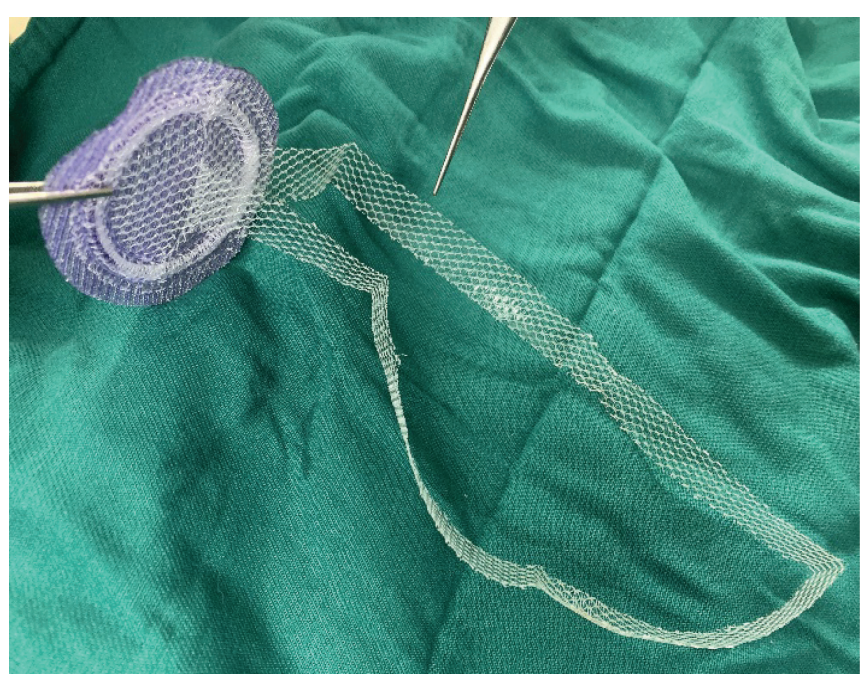

Figure-2 Self-expandable dual mesh with two tails 


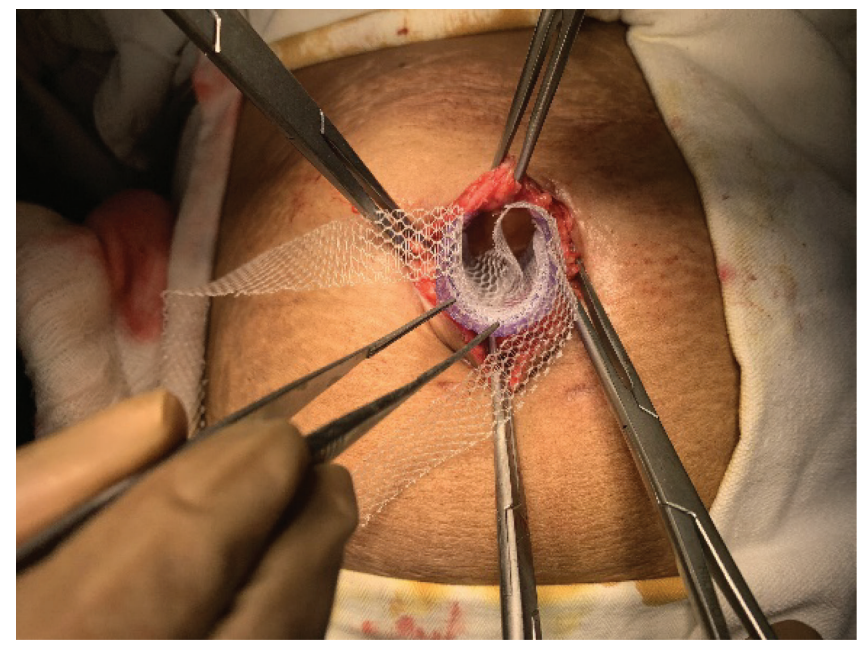

Figure-3 : (a) Ventralex mesh is folded and inserted

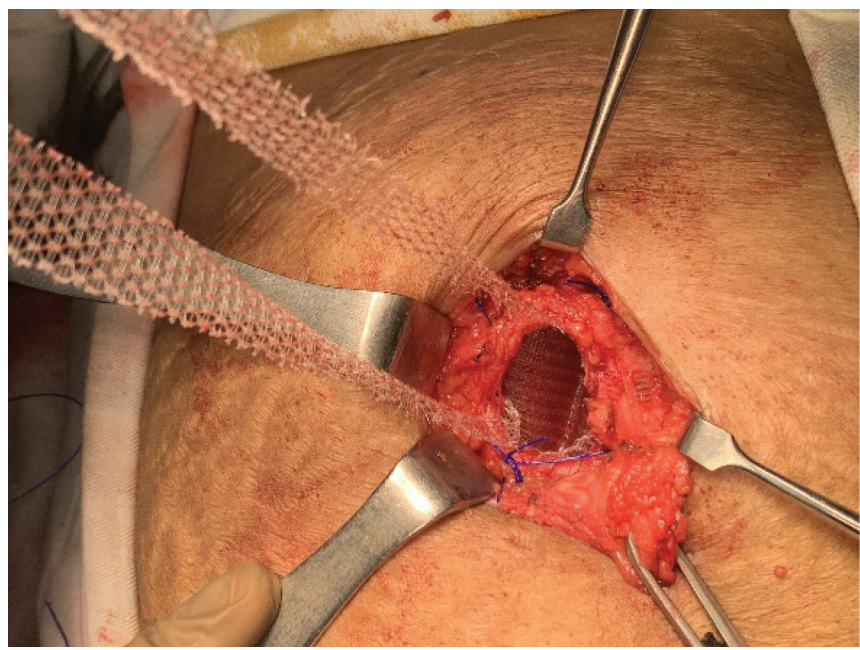

Figure-3 (b) Fixation of the mesh intraperitonealy

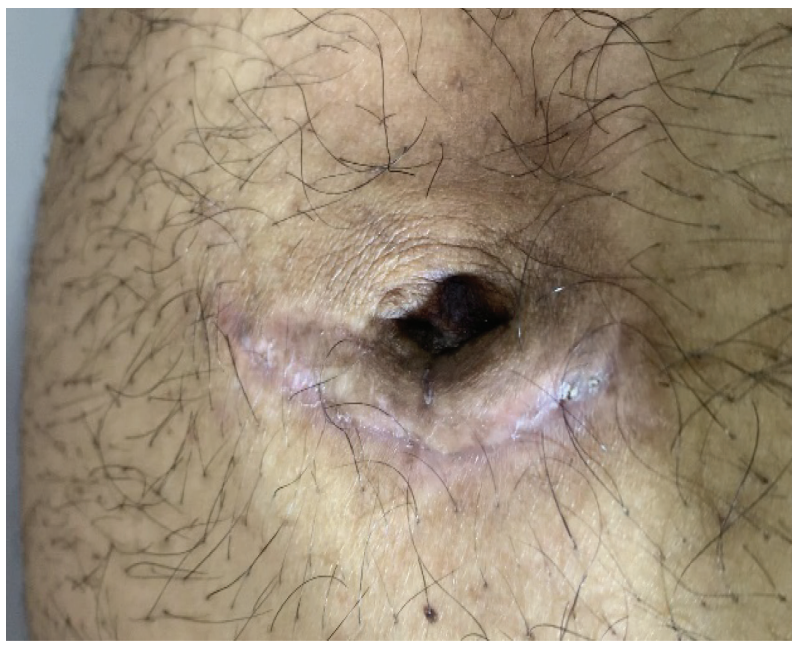

Figure-4 (a) : After 6 weeks

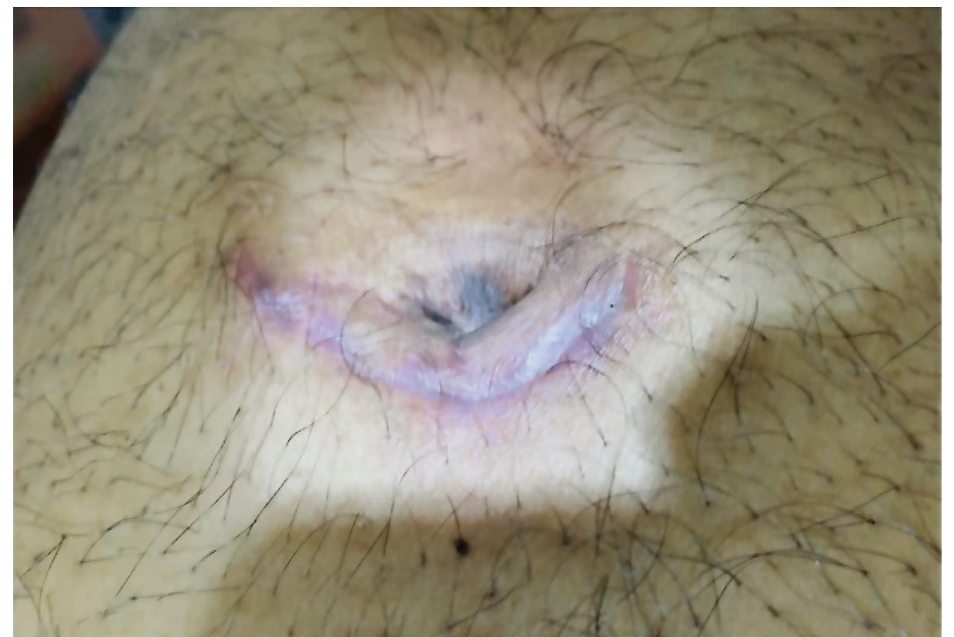

Figure-4 (b) : 1 year follow up view

\section{Patient follow-up and evaluation of patient satisfaction}

Post-operative visits were at first week and sixth week according to the routine follow up, patients had subsequent follow-up organized if deemed necessary. The interviews of all the patients were made at 6 months and 12 months post operatively via telephone. Inquiries consisted of any adverse event related to the procedure, including hernia recurrence and return of symptoms such as pain, discomfort or swelling.

Chronic pain was assessed using the visual analogue score (VAS) and quality of life (QoL) using a comprehensive scoring system (Carolinas comfort scale; CCS) specially designed for hernia repairs. ${ }^{27}$ Overall satisfaction of patients regarding the surgery and outcome was also assessed and dissatisfied patients were offered a follow-up consultation with the operating surgeon at any point of their follow up. (Figure-4)

\section{Results}

In our study, there were 28 female and 8 male patients with the age ranging from 24-68 years (Mean $41.5 \pm 8.36$ ). Most of them (27) had umbilical hernias with no history of previous abdominal surgery and remaining had umbilical port hernias. (Table-I)

The size of the gap was ranging from 0.8 to $3 \mathrm{~cm}$ as determined by the ultra-sonogram. No major short-term complications were found but minor early complications were in 5 patients (13.89\%).(Table-II) No pain or mild pain was reported in 31 patients after one week's post-operative follow up. 35 patients reported being very satisfied with their repairs. (Table-III) 
Table-I

Demographics, Clinical and Mesh Characteristics. (n=36)

\begin{tabular}{|l|c|}
\hline Demographic Data & Number of patient(N)- 36 \\
\hline - $\quad$ Male/Female Ratio & $28 / 8$ \\
\hline - $\quad$ Age mean (range) & $41.5 \pm 8.36(24-68)$ \\
\hline \multicolumn{2}{|l|}{ Clinical characteristics of hernia } \\
\hline - Umbilical hernia & 27 \\
\hline - Umbilical Port hernia & 9 \\
\hline Mesh Characteristics & \multicolumn{2}{|l}{} \\
\hline$-\quad 6.4 \mathrm{~cm}$ mesh & 15 \\
\hline$\bullet \quad 8 \mathrm{~cm}$ mesh & 21 \\
\hline
\end{tabular}

Table-II

Summary of Complications $(\mathrm{N}=\mathbf{0 5})$

\begin{tabular}{|l|c|}
\hline Type of Complication & Number (\%) \\
\hline i) Early Complications & $5(13.89 \%)$ \\
\hline Superficial Wound Infection & $2(5.56 \%)$ \\
\hline Seroma Formation & $1(2.78 \%)$ \\
\hline Serosanguinous discharge & $1(2.78 \%)$ \\
\hline Bruise/Ecchymosis & $1(2.78 \%)$ \\
\hline ii) Late Complications & $1(2.78 \%)$ \\
\hline Hypertrophied Scar & $1(2.78 \%)$ \\
\hline
\end{tabular}

There were actions taken regarding 2 patients with dressing and antibiotic. For another 4 patients, actions were taken with conservative, regular dressing, conservative, topical steroid respectively.

\section{Table-III}

Analysis of Postoperative pain and Overall satisfaction by quality of life assessment $(n=36)$.

\begin{tabular}{|l|ccc|}
\hline VAS (Visual Analog score in $\mathrm{cm})$ & 1 month & 6 month & 12 month \\
\hline No pain $(<0.5 \mathrm{~cm})$ & 31 & 34 & 36 \\
\hline Mild pain $(>0.5$ to $<4.5 \mathrm{~cm})$ & 4 & 1 & 0 \\
\hline Moderate pain $(>4.5$ to $<7.5 \mathrm{~cm})$ & 1 & 0 & 0 \\
\hline Severe pain $(>7.5 \mathrm{~cm})$ & 0 & 0 & 0 \\
\hline CCS (Carolinas comfort score) & & & \\
\hline Very Satisfied $(<0.05)$ & 32 & 34 & 35 \\
\hline Satisfied $(>0.5$ to $<0.3)$ & 3 & 1 & 0 \\
\hline Neutral $(>0.3$ to $<0.6)$ & 1 & 1 & 1 \\
\hline Not Satisfied $(>0.6)$ & 0 & 0 & 0 \\
\hline
\end{tabular}

*Visual Analog score in cm. ${ }^{* *}$ Carolinas comfort score- Calculated by;

\section{Discussion}

We studied retrospectively 36 patients who underwent mesh repair surgery by the Ventralex ${ }^{\mathrm{TM}}$ hernia patch between April 2017 and October 2019 at Anower Khan Modern Medical College \& Hospital, Care Medical College \& Hospital and LABAID Specialized Hospital. In our study, there were 28 female and 8 male patients with the range of age from 24-68 years (Mean 41.5 \pm 8.36 ) having 27 umbilical hernias and 9 umbilical port hernias.

In this study we found that the Ventralex ${ }^{\mathrm{TM}}$ hernia patch is an effective and easier technique for mesh repair in umbilical and umbilical port hernias. This technique can also save the operative time with less post-operative complications and better outcomes. In several studies ${ }^{23,25,28}$ meshes were used for abdominal wall defects $<1 \mathrm{~cm}$ in diameter, including large sized VentralexTM patches in $>50 \%$ of cases in one series. ${ }^{23}$ Trying to insert a mesh via any such small opening is in all likelihood to rupture it's memory recoil ring, accordingly compromising the fulfillment of the repair because the patch will now not lie flat in opposition to the abdominal wall. It is likewise not possible to reap direct tactile feedback via this type of small defect at some stage in mesh placement so right deployment of the patch can't be ensured.

In our study we did not consider the use of reinforcement prosthesis for the repair of abdominal wall defects $<1 \mathrm{~cm}$ in diameter. Even though all of our patients were routinely discharged from hospital on prophylactic oral antibiotics, two of them $(5.56 \%)$ developed superficial wound infection that was successfully treated with oral antibiotics. Abdominal wall and mesh infection are known risk factors for early hernia recurrence and sometimes require prosthesis removal, especially when containing ePTFE. ${ }^{29}$

Ventral and in particular umbilical hernia repairs are associated with a higher rate of infection of up to $20 \% .{ }^{30}$ Beside older age and comorbidities, wound infection may relate to skin devascularisation when creating the umbilical skin flap or as a consequence of normal umbilical bacterial colonisation. Therefore, we think that soaking the mesh in Gentamicin prior to its insertion and closing the anterior fascia at the end of the procedure will minimize the risk of developing deep wound and mesh infection, as well as the aforementioned risk of recurrence. Studies utilising other type of mesh have also found that fascia closure was associated with lower infection (31) and hernia recurrence ${ }^{32}$ rates. Since completion of this study we have now replaced the original VentralexTM hernia patch by a newer and theoretically safer version of the prosthesis in terms of infection, as the ePTFE side of the dual-mesh has been replaced by an absorbable hydrogel 
barrier based on the same technology used in Seprafilm ${ }^{\circledR}$ (VentralexTM ST hernia patch, Bard ${ }^{\circledR}$, Davol, Warwick, RI). Following their surgery, our 31 interviewed patients did not experience any residual pain, defined as a VAS score of 0/10. Furthermore, 35 patients reported being satisfied or very satisfied with their hernia repair as assessed by the CCS. In comparison, Tollens et al. ${ }^{25}$ mentioned a significant number of patients who experienced a painful sensation when wearing tight clothing (12\%) and/or complaint of a foreign-body type sensation (5\%). Iversen and colleagues ${ }^{24}$ only reported 'chronic pain' in $1.3 \%$, but the calculated VAS scores were above $0 / 10$ in many of their patients. Moreover, $17.9 \%$ of their patients indicated deterioration in their self-estimated general health. Many clinicians define chronic pain as a pain lasting for more than 3 months despite the fact that the injury has healed. This definition is too broad, unclear and makes it therefore difficult to objectively compare results arising from different studies.

\section{Conclusion}

In our study, we believe that that The Ventralex ${ }^{\mathrm{TM}}$ hernia patch is an effective and easier technique for mesh repair in umbilical and umbilical port hernias. The surgeons may be abided by these recommendations to achieve similar outcomes: using this approach for small defects $1-3 \mathrm{~cm}$ in diameter, avoiding using a large-sized patch, fixing the positioning straps with minimum tension, always closing the fascia defect and placing the mesh in the extraperitoneal space (sublay) wherever possible. Finally we found that the Ventralex ${ }^{\mathrm{TM}}$ hernia patch is an effective and easier technique for mesh repair in umbilical and umbilical port hernias and also saves operative time with less post-operative complications and better outcomes.

\section{References}

1. Xie $Y$, Song $Y$, Jian F. Retrospective analysis of smaller than $3-\mathrm{cm}$ umbilical hernia repair with the lightweight macroporous mesh. Medicine. 2018; 97:1-5

2. Burger JW, Roland XX, Luijendijk RW. Long-term follow-up of a randomized controlled trial of suture versus mesh repair of incisional hernia. Ann Surg. 2004; 240:578-585

3. Berrevoet F, Van den BB, de Baerdemaeker L. Laparoscopic evaluation shows deficiencies in memory ring deployment during small ventral hernia repair. World J Surg. 2010; 34:1710-1715

4. Halm JA, Heisterkamp J, Veen HF. Long-term follow-up after umbilical hernia repair: are there risk factors for recurrence after simple and mesh repair. Hernia. 2005; 9:334-7

5. Tollens T, Hondt MD, Devroe K. Retrospective analysis of umbilical, epigastric, and small incisional hernia repair using the Ventralex hernia patch. Hernia. 2011; 15:531-40

6. Sanjay P, Reid TD, Davies EL. Retrospective comparison of mesh and sutured repair for adult umbilical hernias. Hernia. 2005; 9:1

7. Iqbal CW, Pham TH, Joseph A, Mai J, Thompson GB. Long-term outcome of 254 complex incisional hernia repairs using the modified RivesStoppa technique. World J Surg. 2007; 31: 2398-2404

8. Christoffersen MW, Helgstrand F, Rosenberg J. Lower reoperation rate for recurrence after mesh versus sutured elective repair in small umbilical and epigastric hernias: A Nationwide Register Study. World J Surg. 2013; 37:2548-52

9. Ambe P, Meyer A, Köhler L. Repair of small and medium size ventral hernias with a Proceed Ventral Patch: a single center retrospective analysis. Surg Today. 2013; 43:381-5

10. Christoffersen MW, Helgstrand F, Rosenberg J, et al. Long-term recurrence and chronic pain after repair for small umbilical or epigastric hernias: a regional cohort study. Am J Surg. 2016; 211:305-6

11. Perrakis E, Velimezis G, Vezakis A, Antoniades J, Savanis $\mathrm{G}$, Patrikakos $\mathrm{V}$ et al. A new tension-free technique for the repair of umbilical hernia, using the prolene hernia system - Early results from 48 cases. Hernia. 2003; 7:178-80

12. Trujillo CN, Fowler A, Al-temimi MH, Ali A, Johna S, Tessier D. Complex ventral hernias: a review of past to present. Perm J. 2018; 22:17-015

13. Reynolds JC, Ward PJ, Rose S, Solomon M. Small bowel. In: Reynolds JC, Ward PJ, Rose S, Solomon M, eds. Netter Collection of Medical Illustrations: Digestive System: Part II - Lower Digestive Tract. 2nd ed. Philadelphia, PA: Elsevier; 2017; 31-114

14. Rodriguez JA, Hinder RA. Surgical management of umbilical hernia. Oper Tech Gen Surg. 2004; 6:156-64

15. Bougard H, Coolen D, Beer R, D Folscher D, Kloppers JC, Koto MZ et al. HIG (SA) Guidelines for the Management of Ventral Hernias. SAJS. 2016; 54:S2-S26.

16. Köckerling F. Onlay technique in incisional hernia repair $\square$ a systematic review. Front Surg. 2018; 5:71

17. George CD, Ellis $\mathrm{H}$. The results of incisional hernia repair: a twelve year review. Ann R Coll Surg Engl. 1986; 68: 185-187.

18. Paul A, Korenkov M, Peters S, Köhler L, Fischer S. Unacceptable results of the Mayo procedure for repair of 
abdominal incisional hernias. Eur J Surg. 1998; 164: 361-367

19. Schumacher OP, Peiper C, Lörken M, Schumpelick V. Long-term results after Spitzy's umbilical hernia repair. Chirurg. 2003; 74: 50-54

20. Millikan KW, Baptista M, Amin B, Deziel DJ, Doolas A. Intraperitoneal underlay ventral hernia repair utilizing bilayer expanded polytetraXuoroethylene and polypropylene mesh. Am Surg. 2003; 69:287-291

21. Hadi HI, Maw A, Sarmah S, Kumar P. Intraperitoneal tension-free repair of small midline ventral abdominal wall hernias with a Ventralex hernia patch: initial experience in 51 patients. Hernia. 2006; 10: 409-413

22. Martin DF, Williams RF, Mulrooney T, Voeller GR. Ventralex mesh in umbilical/epigastric hernia repairs: clinical outcomes and complications. Hernia. 2008; 12: 379-383

23. Berrevoet F, D'Hont F, Rogiers X, Troisi R, de Hemptinne B. Open intraperitoneal versus retromuscular mesh repair for umbilical hernias less than $3 \mathrm{~cm}$ diameter. Am J Surg. 2011; 201: 85-90

24. Iversen E, Lykke A, Hensler M, Jorgensen LN. Abdominal wall hernia repair with a composite ePTFE/polypropylene mesh: clinical outcome and quality of life in 152 patients. Hernia. 2010; 14: 555-560

25. Tollens T, Den HM, Devroe K, Terry C, Speybroeck S. Retrospective analysis of umbilical, epigastric, and small incisional hernia repair using the VentralexTM hernia patch. Hernia. 2011; 15: 531-540
26. Berrevoet F, Van den Bossche B, de Baerdemaeker L, de Hemptinne B. Laparoscopic evaluation shows deficiencies in memory ring deployment during small ventral hernia repair. World J Surg. 2010; 34: 1710-1715

27. Heniford BT, Walters AL, Lincourt AE, Novitsky YW, Hope WW. Comparison of generic versus specific quality-of-life scales for mesh hernia repairs. J Am Coll Surg. 2008; 206: 638-644

28. Tinelli A, Malvasi A, Manca C, Alemanno G, Bettocchi S. Postlaparoscopic mesh in post-menopausal umbilical hernia repair: a case series. Minim Invasive Ther Allied Technol. 2011; 20: 290-295

29. Stremitzer S, Bachleitner-Hofmann T, Gradl B, Gruenbeck M, BachleitnerHofmann B. Mesh graft infection following abdominal hernia repair: risk factor evaluation and strategies of mesh graft preservation: A retrospective analysis of 476 operations. World J Surg. 2010; 34:1702-1709

30. Farrow B, Awad S, Berger DH, Albo D, Lee L. More than 150 consecutive open umbilical hernia repairs in a major Veterans Administration Medical Center. Am J Surg. 2008; 196: 647-651

31. Petersen S, Henke G, Zimmermann L, Aumann G, Hellmich G. Ventral rectus fascia closure on top of mesh hernia repair in the sublay technique. Plast Reconstr Surg. 2004; 114: 1754-1760

32. Booth JH, Garvey PB, Baumann DP, Selber JC, Nguyen AT. Primary fascial closure with mesh reinforcement is superior to bridged mesh repair for abdominal wall reconstruction. J Am Coll Surg. 2013; 217: 999-1009 